\title{
Industrial Environmental Monitoring With Wireless Data Storage and Manipulation System
}

\author{
Keerthika $\mathrm{K}^{1}$, Amudhasurabhi $\mathrm{P}^{2}$, Balamurali $\mathrm{V}^{3}$, Beryl Georgina $\mathrm{R}^{4}$ \\ ${ }^{1}$ Assistant professor, Department of ECE, Jerusalem College of Engineering \\ ${ }^{2,3,4}$ UG Scholar, Department of ECE, Jerusalem college of Engineering
}

\begin{abstract}
Anenvironmental monitoring with wireless data storage and manipulation system based on sensors, Wi-Fi (Wireless Fidelity)moduleand internetis designed to keep track of the industrial atmosphere and sensors for measuring temperature, pressure, humidity, and CO are employed in the system to gather information from the industrial environment. The lower layer field data collections system is linked with the upper layer monitoring via Wi-Fi. The monitoring system is implemented with an user friendly interface. The system could store and manipulate gathered information efficiently. Both the software and hardware implementation are demonstrated in this work.
\end{abstract}

Keywords:Environmental monitoring; Wi-Fi; Sensor; the Internet of Things

\section{Introduction}

The Internet of Things (IoT) is an emerging global Internetbased information architecture facilitating the exchange of goods and services in global supply chain networks. From a technical point of view, the architecture is based on data communication tool of sensors and Internet. The sensor nodes can sense, measure, and gather information from the environment and, based on some local decision process, they can transmit the sensed data to the user. The technology of the Internet of things used to sense the information becomes a new research direction of remote monitoring system. Employing sensors, any area could be monitored at anytime. Benefit from the development of sensors, the environmentalmonitoring system could get the data of the target surrounding areas. Then, the collected data is transmitted to the remote monitoring system through Wi-Fi module. Because of its application potential, the environmental monitoring system based on sensors attracts a lot of interests recently. Different systems have been designed for applications, such as manufacture monitoring, water monitoring, mine monitoring, etc. In most of the existing systems, the data is collected by a data collecting module embedded in the target equipments. Then the data can be transmitted through industry Ethernet or wireless networks with Zig-Bee, GPRS (General Packet Radio Service). However, a lot of aged equipments employed in industry don't have embedded data collecting module and network interface. Thus, it is quite difficult to obtain the vital information (temperature,pressure, humidity, and COetc) from these aged equipments. Anenvironmental monitoring system based on sensors is designed thereupon. Sensors are employed to collect data. Wi-Fi (Wireless Fidelity) is used to transmit the data in our designed system. Wireless fidelity includes IEEE $802.1 \mathrm{la} / \mathrm{b} / \mathrm{g}$ standards for wireless local area networks. It allows users to surf the Internet at broadband speeds when connected to awireless access point (WAP) or in ad hoc model. Section II demonstrates the design of software and hardware. The implementation of the system can be found in Section III, and Section IV is the conclusion.

\section{A. Field Layer Design}

\section{Design of Software And Hardware}

The field layer could be parted into two units, such as the data collecting unit and the data-transmitting unit. The vital data of equipments, like temperature, pressure, humidity, and CO (carbon monoxide)could be collected by the data collecting unit. Then the data is transmitted by the data-transmitting unit to the upper layer through Wi-Fi with TCP/IP (Transmission Control Protocol/Internet Protocol). 


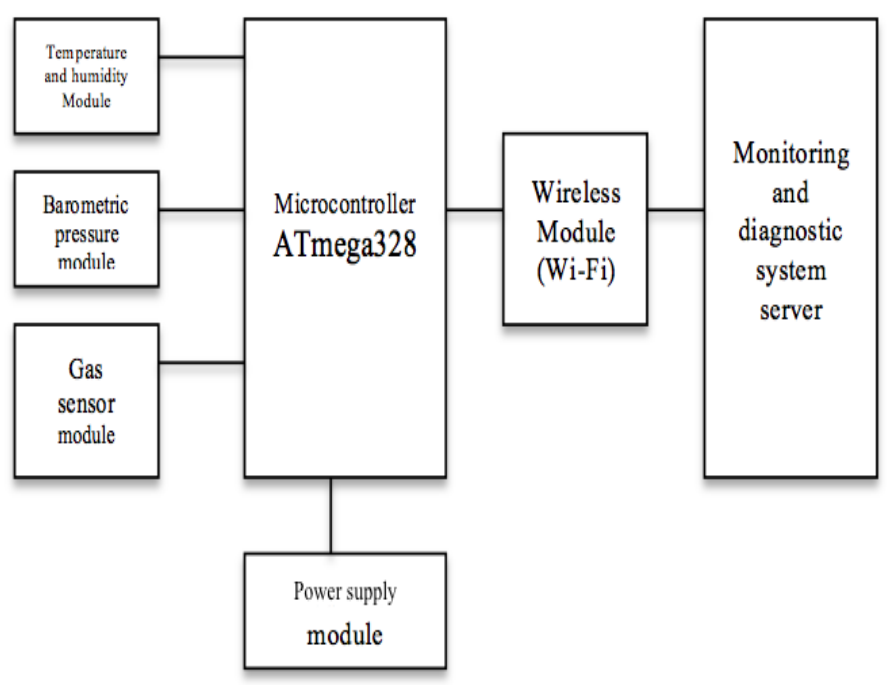

Figure2.1 Construction of the system

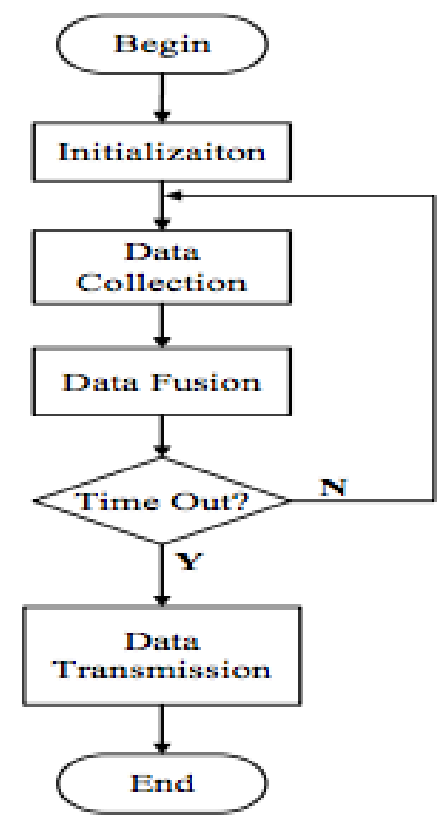

Figure2.2 DataflowDiagram

As depicted inFigure 2.1, the system is constructed by 7 parts,such as: the main control chip ATmega328, the temperature and humidity module, barometric pressure module, the power supply module and the wirelessWi-Fi module.

\section{B. Software Design}

The data from the lower layer field of the system, such as temperature,pressure, humidity, and CO (Carbon monoxide) can be transmitted to the remote terminal supervisor through wireless communication system. An SQL (Structured Query Language) database is constructed to keep the collected data. The software of the system includes data collection, transmission, display and analysis. The data collection includes the data process in sensors and host microcontroller. Data transmission includes the communication between host microcontroller and monitoring and diagnostic system server based on Wi-Fi. The data collection and transmission processing is described as follows.

Step 1: Initializing system;

Step 2: Collecting data from the sensors, such as temperature,pressure, humidity, and CO (Carbon monoxide). The Analog signals are converted to digital signals by A/D converter in the host microcontroller;

Step 3: Data Preprocessing and data fusion in host microcontroller; 
Step 4: Periodically Sending Data to the monitoring and diagnostic system server. To compatible communication technology Wi-Fi, the communication software is programmed based on TCP/IP protocol.

\section{A. Remote Monitoring Hardware Implementation}

\section{Implementation}

A picture of the data collecting module and the transmitting module is shown in Figure 3.1. Different sensors, such as, temperature and humidity transducer, pressure transducer and a gas transducer are employed here. Wi-Fi is used to transmit the information from monitoring module to the upper layer.

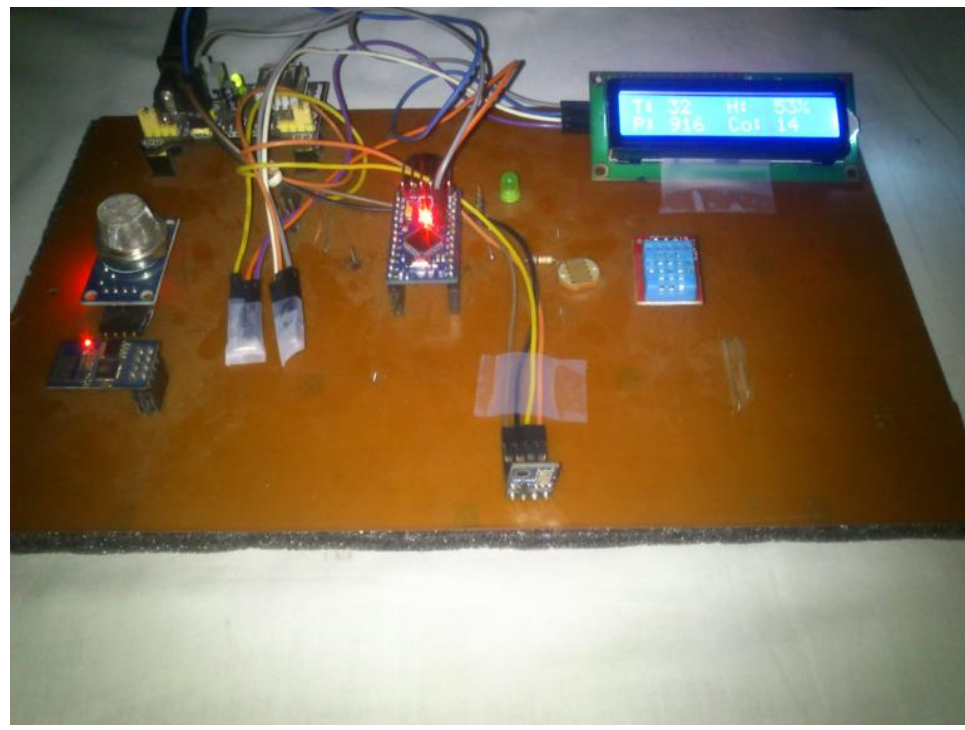

Figure 3.1 Monitoring Kit

\section{B. Remote Monitoring Software Implementation}

The testing system includes different functions, such as real time display, alarm, historical data query, data analysis, etc. In this system, the data can be published to the browser within two seconds. Meanwhile, the data is analyzed by the system. Consequently, remote users could monitor the vital data of target equipment through Internet. Then remote manipulation could be achieved based on the monitoring system. The software interface is demonstrated in the web form, where four environmental parameters are monitored. It is easy to expand the system to more parameters.

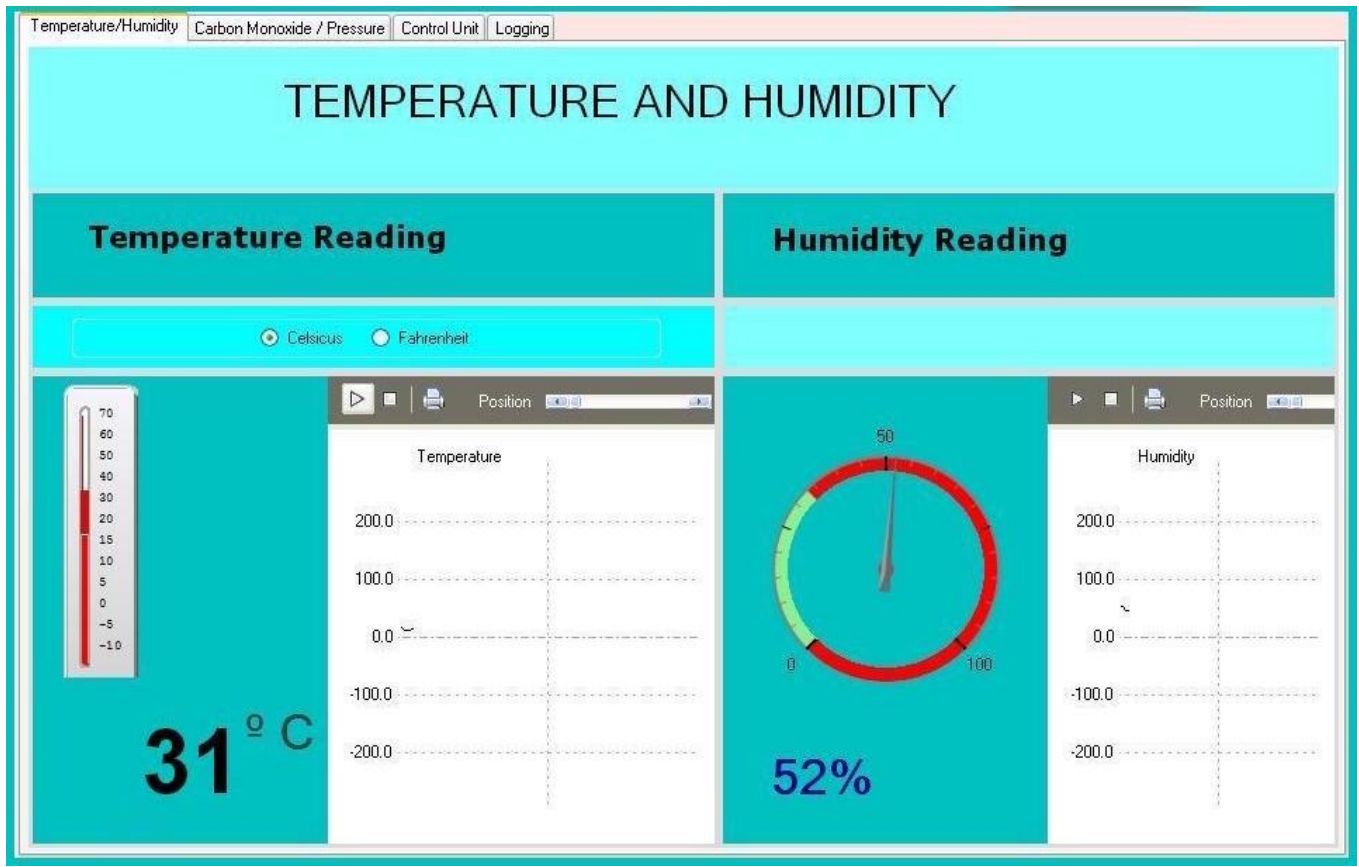

Figure 3.2 Output of temperature and humidity in computer 


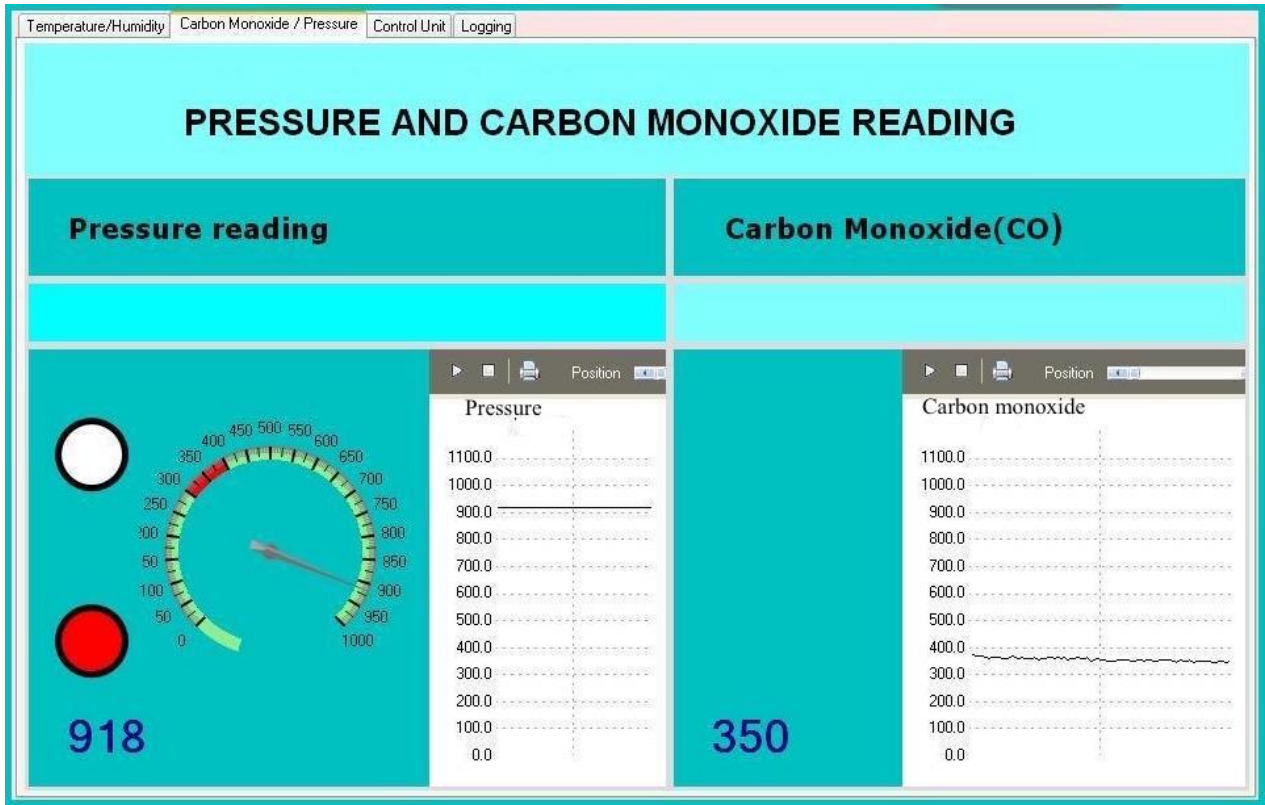

Figure 3.3 Output of pressure and carbon monoxide in computer

\section{Conclusion}

An environmental monitoring with wireless data storage and manipulation system based on sensors, Wi-Fi (Wireless Fidelity) module and Internetis designed in this paper, and themonitoring system is implemented on environmental parameters. Thesystem collects data (temperature, pressure, humidity, and CO) from the sensors, does dataPre-processing and fusion, and sends the data to monitoringServer periodically. The tests show that the system couldmonitor environmental parameters status efficiently.

\section{References}

[1]. J. C. St. John and W. L. Chameides, -Environ. Science Techno 31॥, pp. 2797-2804, 1997.

[2]. IST, Chapter 1-Introduction - International Sensor Technologyll, pp.1-4.

[3]. D. D. Lee and D. S. Lee, —Environmental gas sensors, IIEEE Sensors J., Vol. 1, No. 3, pp. 214-215, 2001.

[4]. NihalKularatna, and B. H. Sudantha, - An Environmental Air Pollution Monitoring System Based on the IEEE 1451 Standard for Low Cost Requirements\| IEEE Sensors Journal, Vol. 8, No. 4, 2008

[5]. Xu, N, -A Survey of Sensor Network Applications, IIEEE Communications Magazine, Vol. 40, No.8, pp. 102-114, 2002.

[6]. Young Jin Jung, Yang Koo Lee, Dong Gyu Lee, Keun Ho Ryu, Silvia Nittel —Air pollution monitoring system based on geosensor network, IEEE International Geoscience and Remote Sensing Symposium, pp.III-1370-III-1373, 2008.

[7]. Electrochemical CO sensors\|, Targeted Wind Sensing.http://tws.unh.edu/index.php?option=com_content\&task=vi ew\&id=63\&Itemid=7 